\title{
Of Open Pits and Valleys: Endoscopic Optical Diagnosis Juxtaposition of Pathologically Distinct Rectal Polyp Lesions
}

\author{
Vincent Zimmer ${ }^{1,2} \quad$ Bert Bier ${ }^{3}$ \\ ${ }^{1}$ Department of Medicine, Marienhausklinik St. Josef Kohlhof, \\ Neunkirchen, Germany \\ ${ }^{2}$ Department of Medicine II, Saarland University Medical Center, \\ Saarland University, Homburg, Germany \\ 3 Insitute of Pathology Saarbrücken-Rastpfuhl, Saarbrücken, \\ Germany
}

J Digest Endosc 2021;12:175-176.
Address for correspondence Vincent Zimmer, MD, Department of Medicine, Marienhausklinik St. Josef Kohlhof, Klinikweg 1-5, Neunkirchen, 66539, Germany (e-mail: vincent.zimmer@gmx.de).

\begin{abstract}
Keywords

- colon cancer surveillance

- colorectal neoplasia

- colonoscopy

- endoscopic diagnosis

- optical diagnosis

- pit pattern

- magnification endoscopy

- serrated lesion

- colorectal adenoma

Optical diagnosis during colorectal cancer screening is instrumental in deciding whether or not to resect colorectal lesions, choose the appropriate technique and to properly communicate with the pathologist. The latter is even more important when it comes to serrated lesions with the latest WHO classification justifying a pathology diagnosis of a serrated lesion with a minimum criterion of characteristic findings in just one crypt, which may only be detectable when adequate sectioning and scrutinization is performed. Here, we present a unique case of comparatively small rectal lesions with typical endoscopic findings warranting a diagnosis of a serrated lesion (open pit pattern) and adenoma (valley sign).
\end{abstract}

\section{Introduction}

Optical diagnosis of diminutive $(<5 \mathrm{~mm})$ and/or small $(<10$ $\mathrm{mm}$ ) rectal lesions is instrumental in day-to-day endoscopy practice to decide on endoscopic resection and its adequate modality. Identification of serrated lesions has been operationalized by the workgroup serrated polyps and polyposis (WASP) classification. In contrast, type II-O pit pattern as dilation crypt opening due to mucus production are only appreciated by specialized techniques, while the so-called "valley sign" signifies a small adenomatous lesion.

\section{Case Presentation}

An 80-year-old male patient presented for colonoscopy for anemia workup. While no distinct recent and/or potential bleeding source was detected throughout the colon, $>15$ mostly small polypoid lesions were resected in the cold snare technique. In addition, two adjacent sessile Paris Is lesions were identified in the middle third of the rectum ( - Fig. 1A). Of interest, these lesions appeared different from one another with the somewhat larger lesion labeled as (a) estimated at $9 \mathrm{~mm}$, revealing small dark spots on its surface
DOI https://doi.org/ 10.1055/s-0041-1739560 ISSN 0976-5042
(C) 2021. Society of Gastrointestinal Endoscopy of India.

This is an open access article published by Thieme under the terms of the Creative Commons Attribution-NonDerivative-NonCommercial-License, permitting copying and reproduction so long as the original work is given appropriate credit. Contents may not be used for commercial purposes, or adapted, remixed, transformed or built upon. (https://creativecommons.org/licenses/by-nc-nd/4.0/). Thieme Medical and Scientific Publishers Pvt. Ltd. A-12, 2nd Floor, Sector 2, Noida-201301 UP, India 


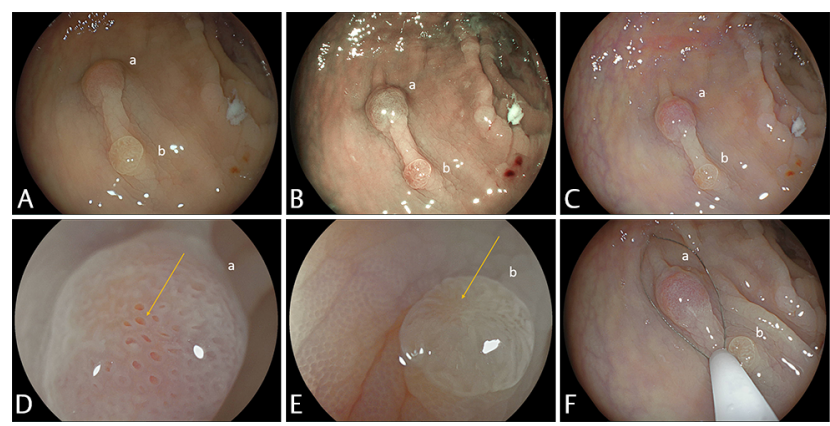

Fig. 1 (A) White-light endoscopy (WLE) identifies two rectal is lesions of (a) $9 \mathrm{~mm}$ and (b) $5 \mathrm{~mm}$, respectively. The larger lesion (a) exhibits signs of a potential serrated lesion among others with fine dark spots on its surface, while the smaller lesion (b) demonstrates a "valley sign" as an adenoma marker with a discrete central depression surrounded by a regular branched surface pattern (JNET type 2A). (B, C) Image-enhanced endoscopy (IEE) using blue-laser imaging (BLI) and linked color imaging ( $\mathrm{LCl}$ ) confirms and extends these findings. (D) Magnification IEE in the $\mathrm{LCl}$ mode clearly depicts type-II open pit pattern (arrow) correlating to widened crypt openings in lesion (a). (E) Likewise, the smaller lesion (b) clearly demonstrates central amorphous with an inconspicuous peripheral surface pattern, the so-called "valley sign" (arrow), not to be mixed up with evidence of advanced histology. (F) The lesions were resected en bloc with wide margins using a cold snare. JNET, Japanese Narrow Band Imaging expert team.

suspicious of a serrated lesion as per the work group serrated polyps and polyposis (WASP) classification. ${ }^{1}$ In the WASP classification, assessment for adenoma characteristics is performed first (brown color and tubular or branched surface). In the absence of these findings, in a next step, the categories cloudiness, indistinctive borders, irregular shape, and dark spots inside crypts (at least, two of four features needed to classify as a serrated over hyperplastic lesion. By contrast, the smaller lesion designated (b) exhibited a discrete, although reproducible central depression with peripheral gyriform regular surface pattern. Image-enhanced endoscopy using blue-laser imaging (BLI; - Fig. 1B) and linked color imaging (LCI; - Fig. 1C) substantiated the optical diagnosis of a serrated lesion (a; analogous to Japanese Narrow Band Imaging expert team [JNET] type 1) and low-grade intraepithelial neoplasia (b; analogous to JNET type 2A). Next, the lesions were characterized by magnification endoscopy in the LCI mode illustrating type-II open pit pattern ( $\boldsymbol{- \text { Fig. }}$ 1D) and the so called "valley sign" as an uncommon and insensitive, though characteristic finding 2,3 ( - Fig. 1E). Both lesions were cold snare resected with wide margins ( $\mathbf{- F i g}$. 1F), and pathology confirmed the optical diagnoses of a serrated adenoma/polyp (SSA/P) without dysplasia ( - Fig. 2A, B) and low-grade tubular adenoma ( - Fig. 2C, D).

Optical diagnosis of diminutive $(<5 \mathrm{~mm})$ and/or small $(<10 \mathrm{~mm})$ lesions is challenging in clinical practice, in particular, when examination settings are suboptimal and concerning, for example, training, cleanliness, appropriate technology, and others. Notwithstanding, the "resect-and-discard" concept potentially to be considered for diminutive polyps $<5 \mathrm{~mm}$ with a confident optical diagnosis

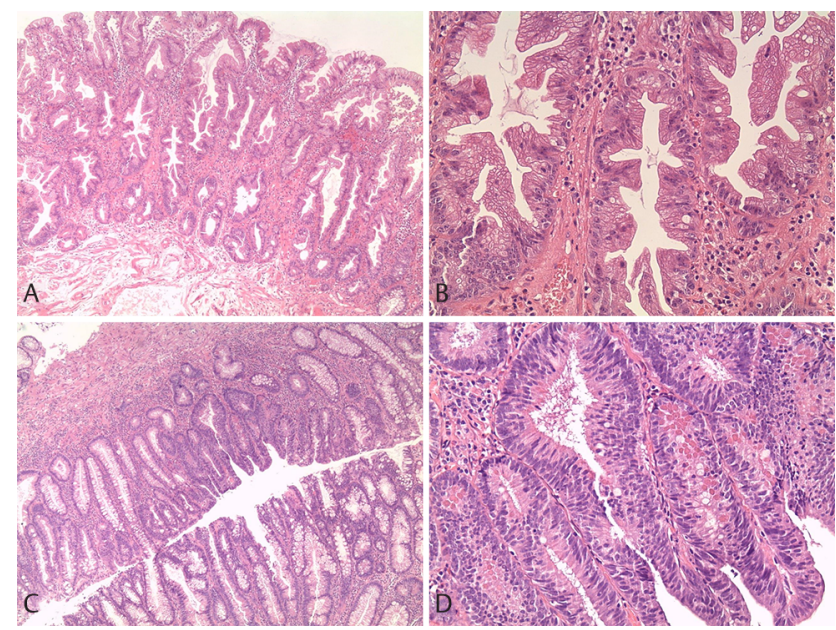

Fig. 2 (A, B) Histopathology of lesion confirming the optical diagnosis of sessile serrated adenoma/polyp (SSA/P) without dysplasia, $\mathrm{H} \& \mathrm{E}, \times 5$ and $\times 20$, respectively. (C, D) Representative histopathology of lesion indicating tubular colorectal adenoma with low-grade intraepithelial neoplasia. $\mathrm{H} \& \mathrm{E}, \times 5$ and $\times 20$, respectively. $\mathrm{H} \& \mathrm{E}$, hematoxylin and eosin.

by a well-trained and committed expert endoscopist has not yet been adopted widely for various reasons. Beyond this "resect-and-discard" discussion, however, given updated World Health Organization (WHO) classification criteria for sessile serrated lesions (SSLs) definition implying pathology detection of only one unequivocal aberrant crypt, adequate input on the endoscopy part into the interaction with pathology appears crucial in a real-world setting, since even the pathology diagnosis of SSLs is easily missed (discrete findings, inadequate number of sections examined, etc.). ${ }^{4}$ In fact, in the presented clinical case, typical pathology findings warranting a diagnosis of SSL was only noted after further sectioning the resection specimen. However, with a view to its precursor nature and accelerated colorectal carcinogenesis, adequate diagnosis and treatment of SSLs are key to high-quality colorectal cancer screening.

\section{Conflict of Interest}

None declared.

\section{References}

1 IJspeert JE, Bastiaansen BA, van Leerdam ME, et al. Dutch Workgroup serrAted polypS \& Polyposis (WASP). Development and validation of the WASP classification system for optical diagnosis of adenomas, hyperplastic polyps and sessile serrated adenomas/polyps. Gut 2016;65(6):963-970

2 Matsushita HO, Yamano HO. What is type II-open pit pattern? Dig Endosc 2016;28(Suppl 1):60

3 Rex DK, Ponugoti P, Kahi C. The "valley sign" in small and diminutive adenomas: prevalence, interobserver agreement, and validation as an adenoma marker. Gastrointest Endosc 2017;85(3):614-621

4 Crockett SD, Nagtegaal ID. Terminology, molecular features, epidemiology, and management of serrated colorectal neoplasia. Gastroenterology 2019;157(4):949-966.e4, e4 